\title{
On Uncertainties in Determination of Sediment Transport Rates in Coastal Regions
}

\author{
Rafał Ostrowski \\ Institute of Hydro-Engineering, Polish Academy of Sciences, Kościerska 7, 80-328 Gdańsk, Poland, \\ e-mail: rafal.o@ibwpan.gda.pl
}

(Received November 30, 2016; revised January 18, 2017)

\begin{abstract}
The paper deals with research uncertainties, difficulties, inaccuracies and unreliabilities related to the modelling of physical phenomena involving coastal lithodynamics. The considerations are focused on processes of wave transformation, wave-driven currents and sediment transport itself. It is shown that possible inaccuracies at individual stages of the modelling of coastal hydrodynamics can lead to serious uncertainties with respect to the ultimate modelling output, namely bed shear stresses and sediment transport rates. These inaccuracies result mostly from arbitrarily assumed parameters and constants. Other modelling biases discussed in the paper comprise simplifications and approximations with respect to sediment resources and size-graded properties, randomness of hydrodynamic impacts, bottom roughness and land-borne factors involved in coastal lithodynamics.
\end{abstract}

Key words: waves, wave-driven currents, bed shear stresses, friction velocity, sediment transport

\section{Introduction}

In the domain of coastal engineering, most problems, aside from wave-current loads on structures, are related to the evolution of the sea bed profile. In general, these sea bed changes are theoretically modelled by means of spatial variability of net sediment transport rates. Sea bed evolution models are very sensitive to the qualitative and quantitative variability of net sediment transport. Hence the need for its precise determination.

Due to practical reasons, the phenomenon of sediment transport in coastal zones is conventionally divided into longshore and cross-shore transport. The longshore current, generated by waves obliquely approaching the shore and breaking in the surf zone, is a driving force of the longshore transport of sediment. A part of the energy (and momentum) of breaking waves is converted into a steady flow. Precise description of this longshore wave-induced flow has been made possible by the development of the radiation stress concept in the late 1960s. Since then, a number of studies have 
been published, with the longshore flow described as generated by the $S_{x y}$ component of the radiation stress. The theoretical model of longshore currents in a multi-bar coastal zone (for multiple wave breaking), as well as comparisons of the model results with experimental data, are given in the book by Szmytkiewicz (2002a). In most of the theoretical models, the longshore sediment transport is assumed to depend on combined wave and current motion. This combined flow of water gives rise to a coupled bed shear stress, which is a driving force for sand movement along the shore. The cross-shore motion of sediments is caused by waves interacting with a wave-driven current called undertow. The undertow can be modelled by the classic approach of Longuet-Higgins, in which the momentum equation in the cross-shore direction, integrated over water depth and wave period, describes the equilibrium between the derivative of the radiation stress $\left(\partial S_{x x} / \partial x\right)$ and the spatial change in the free-surface slope (resulting from phenomena known as the set-down and the set-up, seawards and landwards from the wave breaking point, respectively). On the other hand, these two components of the momentum equation are in local imbalance at particular depths in the water column. This is because the component containing the water slope is constant over water depth, whereas the radiation stress $S_{x x}$ is variable, which results from the decrease of wave orbital velocities towards the sea bed. This imbalance, which is particularly significant in the surf zone, is the driving force behind the resultant offshore current, known as the undertow. In addition, there is an onshore discharge of water between the wave crest and trough, related to the so-called wave drift (or Stokes drift). As a result of the continuity equation, this onshore current requires compensation in the form of the undertow, see Szmytkiewicz (1996, 2002a, 2002b) for more details. Interaction between waves and the undertow gives rise to resultant sediment transport, the direction of which is principally dependent on a very delicate imbalance between the undertow and an onshore flow caused by the vertical asymmetry of the wave shape.

Sediment transport characteristics are thus very sensitive to hydrodynamic phenomena. The relationships between the driving forces and the ultimate morphological responses are highly non-linear and ought to be investigated thoroughly. First, it should be pointed out that in the cross-shore domain even a small change of proportions between the wave asymmetry effects and the undertow can result in a huge quantitative as well as qualitative modification of the net sediment transport rate. Secondly, real coastal hydrodynamic processes are random. This irregularity is generally expressed in the model by the representative wave height $H$ and period $T$, which is a rough approximation. Within such an approach, the prediction of coastal morphodynamics does not account for all components in random wave series. Particularly, the role of extreme waves in the wave energy spectrum can be underestimated. On the other hand, the representative wave parameters are conventionally applied in sediment transport computations for natural conditions. The wave peak period $T_{p}$ is commonly used, while the representative wave height is assumed in many models as either the root-mean-square wave height $H_{r m s}$ or the significant wave height $H_{s}$. In the study by 
Kaczmarek and Ostrowski (1996) it was deduced that their sediment transport model yields proper results for natural (irregular) wave conditions if $H_{r m s}$ and $T_{p}$ are assumed as the representative input. Certainly, this cannot be guaranteed for all types of wave spectra.

Studies reported in the monograph by Ostrowski (2004) confirmed to a large extent some previous findings published, for example, by Van Rijn et al (2003). It was generally concluded by Ostrowski (2004) that a modelling system showing good results for coastal hydrodynamics does not automatically yield satisfactory output for morphodynamics. However, even the accuracy of hydrodynamic modules is often unacceptably low. Errors in wave modelling can cause further errors in the modelling of wave-induced currents. On a second front, there are errors in the modelling of relations between the wave motion and currents. Van Rijn et al (2003) claim that the classical wave transformation models have the inaccuracy of 10-15\% while for the modelling of longshore and cross-shore currents this inaccuracy amounts to 30-50\% or even more. Thus, the hydrodynamic input into sediment transport models and morphodynamics is already erroneous.

In view of the above, it appears obvious that one can encounter research uncertainties on many fronts and with respect to great many aspects of coastal engineering. Identification and analysis of the most important sources of these doubts, inaccuracies and unreliabilities is the objective of the present paper.

\section{Hydrodynamics}

In non-tidal coastal zones, wave transformation plays a key role in hydrodynamics. Aside from processes such as diffraction, refraction, shoaling and reflection, in which wave energy is conserved, waves are subject to energy loss. Wave transformation on a mildly inclined nearshore bottom, typical of the south Baltic shores, is dominated by wave energy dissipation, mostly due to breaking. Several models reproducing this process have been elaborated and successfully tested with experimental data.

For instance, the wave transformation model developed by Szmytkiewicz (2002a) is based on the phase-averaged approach. Under the assumption of mutually parallel isobaths, the model is capable of determining wave parameters along a multi-bar cross-shore profile. The wave transformation process with multiple wave breaking can be modelled for an arbitrary angle of deep-water waves. In the computations, following Battjes and Janssen (1978), it is assumed that the waves are random and their heights in the entire coastal zone can be described by a Rayleigh distribution. On the basis of experimental observations and other available data, it may be deduced that this rough assumption can lead to inaccuracies of no more than $10 \%$ in the determination of wave heights in the nearshore zone. The so-called "roller effect" is also considered. This means that the wave breaking phenomenon is accompanied by the appearance of a rotating mass of water on the crest of the breaking wave (see Fig. 1). 
According to this concept, the wave energy lost during wave breaking induces the roller.

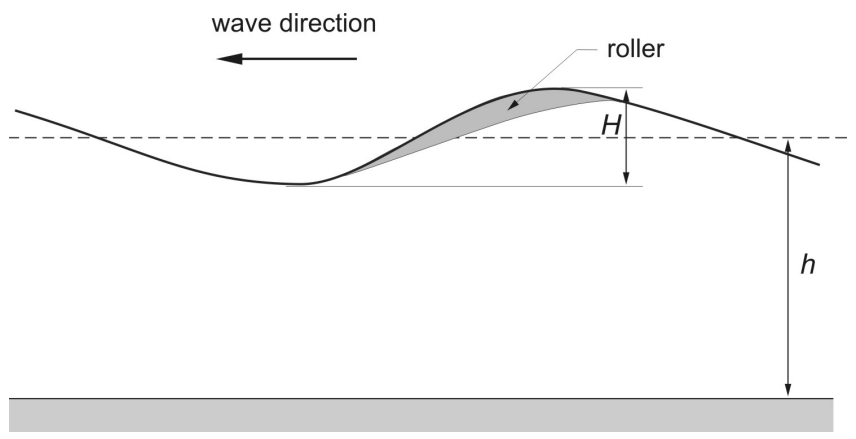

Fig. 1. A roller generated by the breaking wave

In the computational framework for wave transformation, assuming linear wave refraction, the variability of the wave approach angle is calculated from Snell's law and the wave number $k$ is determined from the dispersion relationship for the linear wave theory. Under the assumption that there are no wave reflections from the shore, the wave height is computed from the equation of the energy flux conservation:

$$
\frac{\partial}{\partial x}\left(E C_{g} \cos \theta\right)+\frac{\partial}{\partial x}\left(E_{r} C \cos \theta\right)=-D
$$

where $E$ is the total wave energy, $E_{r}$ is the kinetic energy of the roller (as described by Svendsen 1984), $C$ and $C_{g}$ are the phase and group velocity of waves, respectively, $\theta$ is the wave approach angle, and $D$ is wave energy dissipation.

In the above equation, which is a simplified form of the wave action equation, the wave energy dissipation $D$ is calculated on the hypothesis that the dissipation is related to the wave breaking process only. Assuming a narrow spectrum of random waves in the coastal zone and a Rayleigh distribution of the wave height, the energy dissipation of breaking waves is described by the formula of Battjes and Janssen (1978):

$$
D=\frac{\alpha_{b}}{4} p_{b} f_{p} \rho g H_{\max }^{2},
$$

where $g$ denotes the acceleration due to gravity, $\rho$ is water density, $\alpha_{b}$ is an empirical coefficient of the order $\mathrm{O}(1), f_{p}$ is the wave spectrum peak frequency $\left(f_{p}=1 / T_{p}\right)$, while the factor $p_{b}$, describing the percentage of broken and breaking waves at a given point in the surf zone, is described by the relationship:

$$
\frac{1-p_{b}}{\ln p_{b}}=-\left(\frac{H_{r m s}}{H_{\max }}\right)^{2},
$$

in which $H_{\max }$ denotes the maximum possible wave height at a given location of the coastal zone, and $H_{r m s}$ is the root-mean-square wave height. 
The wave height $H_{r m s}$ is obtained from the system of Eqs. (1), (2) and (3). The maximum possible wave height $H_{\max }$ at a given water depth $h$ in the coastal zone is defined by a criterion formulated by Miche:

$$
H_{\max }=0.88 k_{p}^{-1} \tanh \left(\frac{\gamma_{b} k_{p} h}{0.88}\right),
$$

where $k_{p}$ is the wave number calculated from the dispersion relationship for the linear wave theory with the wave spectral peak $f_{p}$, and $\gamma_{b}$ is an empirical coefficient of wave breaking.

The computed distribution of the wave height $H_{r m s}$ on the cross-shore profile greatly depends on the values of the empirical coefficients $\alpha_{b}$ and $\gamma_{b}$, involved in Equations (2) and (4), respectively. According to the discussion presented by Szmytkiewicz (2002a), the coefficient $\alpha_{b}$ is related to the coefficient $\gamma_{b}$, and, in the calibration of the wave transformation model, $\alpha_{b}=1$ can be assumed and the value of $\gamma_{b}$ is sought only.

Determination of the coefficient $\gamma_{b}$ is associated with the problem of the maximum wave height which can occur under specific local natural conditions. At big water depths, this maximum wave height depends on the wave steepness only, while in the nearshore regions it is influenced by water depth. Theoretically derived from Miche's criterion of wave breaking (see Szmytkiewicz 2002a), the coefficient $\gamma_{b}$ equals 0.88 . However, measurements carried out for both regular and irregular waves imply that this value is much smaller. Based on his own studies (including laboratory and field experiments) and literature review, considerations of Szmytkiewicz (2002a) suggest that the wave breaking coefficient $\gamma_{b}$ can lie in a quite wide range, from 0.3 to 0.8 . For the south Baltic coastal areas, on the basis of field measurements conducted at the IBW PAN Coastal Research Station (CRS) in Lubiatowo, he recommends the application of formula proposed by Battjes and Stive (1985) or assumption of $\gamma_{b} \approx 0.8$. The parameter $\gamma_{b}$ is definitely site-specific, depending on the local wave climate (offshore wave parameters and spectral features), as well as the shape and inclination of the coastal bottom profile.

The study site of CRS Lubiatowo is characterised by a multi-bar sea bottom in the nearshore zone. Such a seabed relief is a typical bottom configuration for the south Baltic sandy coast. Here, except for cliff shores and intensively eroded sandy shore segments, the shape of the coastal sea bed creates favourable conditions for gradual wave energy dissipation due to breaking over consecutive bars, from the offshore region to the shoreline. The above compound process was observed during wave measurements in numerous field surveys. Recently, it has been reproduced theoretically with a satisfactory accuracy by two models, namely the IBW PAN phase-averaged wave transformation model (described above) and the approach based on the hydraulic jump model (HJM), developed at the National Taiwan Ocean University, see Hsu et al (2016). Exemplary results of computations carried out using these models are shown in Fig. 2. Because the HJM is suitable for the surf zone only, the computational region for this model has been specified from the first breaking point to the shoreline. The 
IBW PAN model results cover a slightly bigger area. It also takes into account the wave set-up, which is visible in the non-zero wave height at the shoreline.

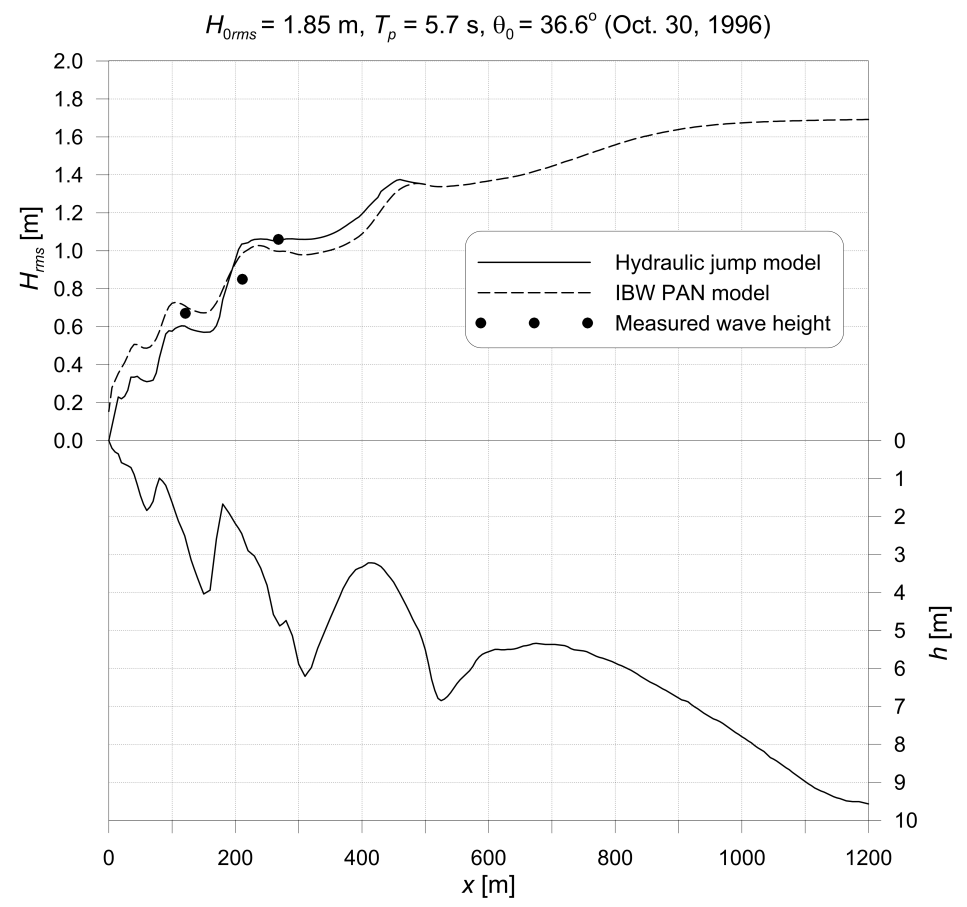

Fig. 2. Wave height modelled using two models and measured at CRS Lubiatowo multi-bar cross-shore profile on $30^{\text {th }}$ October 1996, exemplary results of Hsu et al (2016)

The model results presented in Fig. 2 show reasonably good agreement with in-situ measurements. In most of the cases considered by Hsu et al (2016), the HJM produced higher waves than the IBW PAN model in the outer part of the surf zone, i.e. from depths of $4-5 \mathrm{~m}$ to depths of $1.5-2 \mathrm{~m}$. The situation was the opposite in the inner (nearshore) part of the surf zone, from depths of about 1.5-2 $\mathrm{m}$ to the shoreline.

From the engineering point of view, underestimation of wave heights by theoretical models is highly undesirable. For instance, the design weight of an individual armour unit in the rubble-mound structure is proportional to the design wave height raised to the third power (see Shore Protection Manual 1984). Therefore, even small inaccuracies in determination of the design wave height result in considerable errors in calculation of the armour block weight. Hence, we have to be very cautious while determining design wave parameters in the surf zone. Application of more than one wave transformation model in such cases is highly recommended. Otherwise, improper structural design can occur.

Uncertain output of the wave transformation model constitutes unreliable input into the models of wave-driven currents. In these models, concerning both long- 
shore and cross-shore water flows, such phenomena as bottom friction and turbulence ought to be considered. In general, bottom friction $\tau$ (bed shear stress) is proportional to water density, squared flow velocity and the friction coefficient $f_{w}$. Elaborated by Szmytkiewicz (2002a), a review of several experimental studies of longshore wave-driven currents in seas worldwide yields $f_{w}$ values varying from 0.005 to 0.089 , which clearly proves that modelled longshore currents are significantly dependent on the assumed friction coefficient. Indeed, Szmytkiewicz (2002a) shows that an increase in $f_{w}$ from 0.01 to 0.02 causes the longshore current velocity to decrease by about $50 \%$. On the basis of the results of field surveys carried out at CRS Lubiatowo, he recommends the application of $f_{w} \approx 0.015$ for the south Baltic coastal regions. It should also be noted that the longshore velocities computed by Szmytkiewicz (2002a) for the wave breaking coefficient $\gamma_{b}=0.6$ are locally more than twice as high as the ones determined for $\gamma_{b}=0.8$.

Turbulent processes in theoretical description of wave-induced longshore flows are mostly represented by the "horizontal" turbulent viscosity $v_{t h}$. This parameter, however, which can be determined by a few formulas, has minor influence on modelled longshore currents. The situation is different for cross-shore currents, where the "vertical" turbulent viscosity $v_{t z}$ is a crucial parameter in theoretical description of the seaward water flow known as the return current or undertow. The value of $v_{t z}$ can be determined in many ways. It is mostly assumed to be constant in the water column or variable, increasing upwards from the sea bed. Szmytkiewicz (2002a) shows that in many cases the choice of one of the above assumptions leads to distinctly different computational results. The model with the constant $v_{t z}$ yields undertow velocities that are more uniform in the water column, with nearbed velocity much smaller than in the case of assumed vertically increasing $v_{t z}$. This can play a key role in the modelling of sediment transport under interaction of asymmetrical waves and undertow.

\section{Sediment Transport}

Sediment transport rates are dependent on the flow velocity $u$ and the sediment concentration $c$. For stationary flows, the total sediment transport rate in the water column stretching from the bottom $(z=0)$ to the water surface $(z=h)$ can be calculated by the following formula:

$$
q=\int_{0}^{h} \bar{u}(z) \cdot \bar{c}(z) d z
$$

in which $\bar{u}(z)$ and $\bar{c}(z)$ denote time-averaged velocity and concentration, respectively.

Under conditions of unsteady flows, e.g. under waves or waves combined with a steady current, the instantaneous sediment transport rate is determined as 


$$
q(t)=\int_{0}^{h} u(z, t) \cdot c(z, t) d z
$$

To determine the net (resultant) rate of sediment motion per unit width $q_{\text {net }}$ $\left[\mathrm{m}^{3} / \mathrm{s} / \mathrm{m}\right]$, necessary in the modelling of sea bed morphodynamics, the instantaneous transport rates ought to be integrated at each location in the coastal zone $(x)$ over the wave period $T$ as follows:

$$
q_{\text {net }}(x)=\frac{1}{T} \int_{0}^{T} q(t) d t .
$$

Then, conventionally, the evolution of the sea bottom profile, i.e. the change in water depth $h$ in time $t$, is determined on the basis of the spatial variability of net sediment transport rates $q_{n e t}$ in the direction of the $x$ axis from the following continuity equation for sediment:

$$
\frac{d h(t)}{d t}=\frac{1}{1-n} \frac{d q_{n e t}(x)}{d x},
$$

where $n$ is the soil porosity while deposited.

\subsection{Advection-diffusion Equation for Sediment}

Sediment transport is typically divided into bedload transport, taking place just above the sea bed and reacting almost instantaneously to the local conditions, and suspended load transport, carried out by the water motion and needing time or space to be picked up or to settle down. The suspended transport reacts indirectly to changes in flow or wave conditions through changes in the concentration field. In an accelerating flow, the concentration is typically lower than the equilibrium concentration, which is the concentration that would occur for stationary and uniform conditions, because the sediment has to be picked up and transported upwards by turbulent dispersion. When the flow decelerates or the waves are reduced, more sediment remains in suspension than the flow can support, so the sediment settles down. Therefore, the 3D distribution of the sediment concentration $c(x, y, z, t)$ must be solved first before one can obtain the transport rates of interest.

The distribution of sediment in suspension is governed by the following 3D advection-diffusion equation (see e.g. Roelvink and Reniers 2012):

$$
\frac{\partial c}{\partial t}+u \frac{\partial c}{\partial x}+v \frac{\partial c}{\partial y}+\left(w-w_{s}\right) \frac{\partial c}{\partial z}-\frac{\partial}{\partial z}\left(\varepsilon_{s} \frac{\partial c}{\partial z}\right)-\frac{\partial}{\partial x}\left(\varepsilon_{h} \frac{\partial c}{\partial x}\right)-\frac{\partial}{\partial y}\left(\varepsilon_{h} \frac{\partial c}{\partial y}\right)=0,
$$

where $u, v$ and $w$ are the flow velocities in the $x, y$ and $z$ directions, respectively, $w_{s}$ is the grain settling velocity, $\varepsilon_{s}$ is the vertical dispersion constant and $\varepsilon_{h}$ is the 
horizontal dispersion constant $\left[\mathrm{m}^{2} / \mathrm{s}\right]$. The parameters $\varepsilon_{s}$ and $\varepsilon_{h}$ represent turbulent mixing and are, in physical sense, very similar to the vertical turbulent viscosity $v_{t z}$ and the horizontal turbulent viscosity $v_{t h}$ (cf. section 2). Equation (9) can be applied in various time and spatial scales, from detailed phase-resolving wave-induced transport to slowly-varying tide-induced transport. The horizontal and vertical dispersion constants $\varepsilon_{s}$ and $\varepsilon_{h}$ must reflect the processes which are not explicitly resolved. These constants tend to be higher as the considered time and spatial scales are larger. For example, in sophisticated models of transport over ripple beds each vortex shed by the ripples is resolved and the transport is dominated by the advection terms while diffusion is less important. Within a much coarser approach, where the ripples are represented by an average roughness, the net advection transport by these turbulent vortices is represented as a gradient-type diffusive flux.

To solve Eq. (9), it is necessary to formulate the bottom boundary condition, that is, to determine the so-called reference concentration, which is a nearbed concentration closely related to the bed shear stress. This concentration, given at a specified height, either associated with the grain size or with the bed roughness, is a source of sediment flux from the bottom into the water column.

In some specific applications, the horizontal variations are much more important than vertical non-uniformities, and a depth-averaged approach is justified. In such a case, the advection-diffusion equation averaged over the depth $h$ can be applied:

$$
\frac{\partial h c}{\partial t}+u \frac{\partial h c}{\partial x}+v \frac{\partial h c}{\partial y}-\frac{\partial}{\partial x}\left(\varepsilon_{h} \frac{\partial h c}{\partial x}\right)-\frac{\partial}{\partial y}\left(\varepsilon_{h} \frac{\partial h c}{\partial y}\right)=S,
$$

in which the source/sink term $S[\mathrm{~m} / \mathrm{s}]$ represents sediment exchange with the bottom.

The quantity $S$ must be considered with particular care. Although a few simple approaches to determination of this source term are available (see e.g. Roelvink and Reniers 2012), one should be aware that the parameter $S$ is a key factor representing sophisticated mechanisms of sediment motion, comprising not only features of the sea bed soil, but also bottom roughness and bed shear stresses.

It should be pointed out that the depth-averaged advection-diffusion equation is useless in modelling the transport of sandy sediments, the concentration of which is highly variable in the water column. The depth-averaged approach is therefore applied in the modelling of very fine (cohesive) sediments only.

The advection-diffusion equations Eq. (9) and Eq. (10), three- and two-dimensional, respectively, describe changes in the concentration of particles suspended in the water column. Laboratory experiments and field observations have shown (see e.g. Kaczmarek and Ostrowski 2002) that sandy sediments in the coastal regions are mostly subject to movement in suspension very close to the bed and in the superficial bed layer - as bedload. These phenomena are not taken into account by Eq. (9) and Eq. (10). 


\subsection{Mechanics-based Modelling}

As mentioned above, the concentration of sand under wave and wave-current impact is very high near the bottom. Developed by Kaczmarek (1999), the mathematical model of bedload transport is based on the water-soil mixture approach, with a collision-dominated drag concept and the effective (or equivalent) roughness height $k_{e}$ (necessary for the determination of bed shear stresses). This roughness is calculated by the approximate formula presented by Kaczmarek and Ostrowski (1996). The collision-dominated bedload granular-fluid region stretches below the theoretical bed level, while the turbulent fluid region extends above it, constituting the contact load layer and the outer flow layer. The outer region of pure suspension is characterised by very small concentrations, where the process of sediment distribution may be considered as a convective and (or) diffusive process. In contrast, the granular-fluid region below the theoretical bed level is characterised by very high concentrations, where the inter-granular resistance is predominant. Such a three-layer sediment transport scheme, successfully applied in the modelling of coastal morphodynamics by Ostrowski (2004), is shown in Fig. 3.

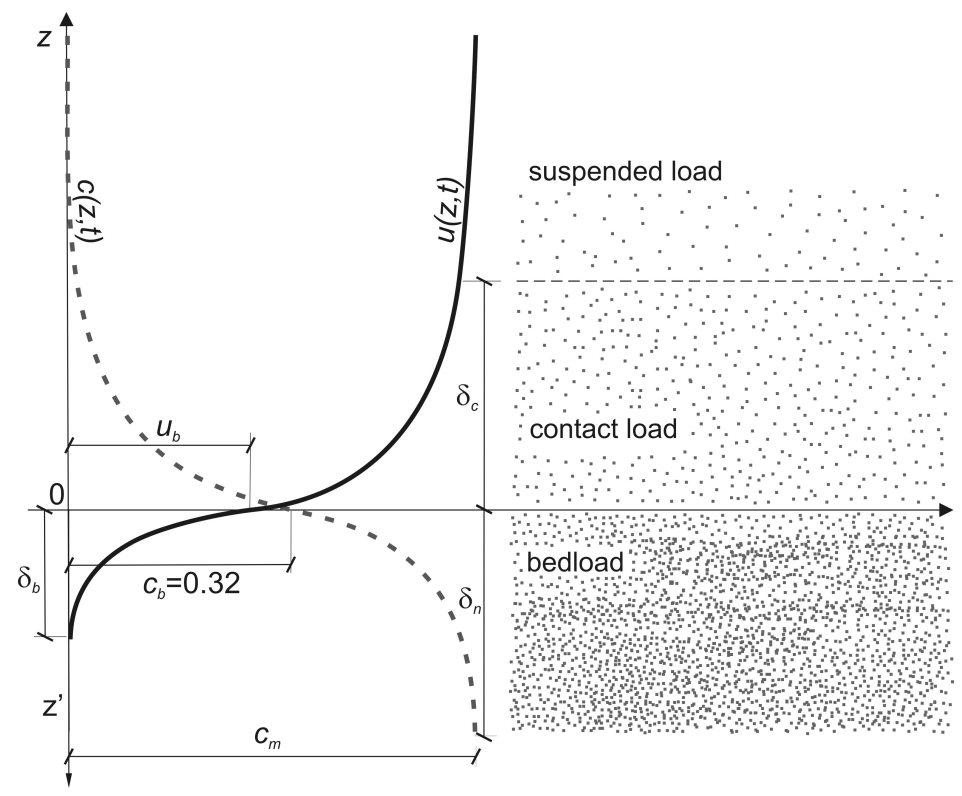

Fig. 3. Three-layer sediment transport model

According to Kaczmarek (1999), the concentration under the bedload transport layer (where the sediment is not moving), at a distance of $\delta_{n}$ below the theoretical bed level, amounts to $c_{m}=0.53$, while at the top of the bedload layer (lower limit of the contact load layer) it is equal to $c_{b}=0.32$. The parameter $c_{b}$ is the sediment concentration corresponding to soil fluidity, while $c_{m}$ is the sediment concentration 
corresponding to the close packing of grains. Both values, $c_{m}$ and $c_{b}$, have significant influence on the solution of the bedload layer. The quantity $c_{m}$, for instance, can lie in a relatively wide range of $0.5-0.77$. The sea bed porosity (implying the concentration of bottom sediments) depends on many factors and circumstances, thus being unpredictable. Therefore, uncertainty about the parameter $c_{m}$ is a drawback of the model.

The velocity profile in the contact load layer is assumed to be continuous. Its intersection with the nominal seabed is the apparent slip velocity $u_{b}$, identified as a characteristic velocity of sediment moving in the form of bedload.

From the hydrodynamic input, described by the nearbed wave-induced oscillatory velocities interacting with wave-driven stationary currents, the instantaneous values of bed shear stresses during a wave period are determined by the momentum integral method proposed by Fredsøe (1984). Then, for known shear stresses, the instantaneous bedload velocities $u\left(z^{\prime}, t\right)$ and concentrations $c\left(z^{\prime}, t\right)$ are computed (with the vertical axis $z^{\prime}$ directed downwards from the theoretical bed level, as defined in Fig. 3). The computations also yield the velocity $u_{b}$ which constitutes input into the solution of the contact load layer. A detailed model description can be found in Kaczmarek (1999) and Kaczmarek and Ostrowski (2002).

The instantaneous values of the sediment transport rate are computed from distributions of velocity and concentration in the bedload layer and in the contact load layer, cf. Eq. (6):

$$
q_{b+c}(t)=\int_{0}^{\delta_{b}} u\left(z^{\prime}, t\right) \cdot c\left(z^{\prime}, t\right) d z^{\prime}+\int_{0}^{\delta_{c}} u(z, t) \cdot c(z, t) d z,
$$

where $\delta_{b}$ is the bedload layer thickness, and $\delta_{c}$ denotes the upper limit of the nearbed suspension (contact load layer thickness). The quantity $\delta_{b}$ results from the solution of the bedload layer, while the value of $\delta_{c}$ is a characteristic bottom boundary layer thickness calculated on the basis of Fredsøe's (1984) approach (see Kaczmarek and Ostrowski 2002).

The net (resultant) transport rate in the bedload and contact load layers is calculated as follows, cf. Eq. (7):

$$
q_{\text {net }}=\frac{1}{T} \int_{0}^{T} q_{b+c}(t) d t .
$$

For the outer flow, due to difficulties in the correct determination of time-dependent concentrations, the net sediment transport rate is calculated in a simplified way, using the time-averaged flow velocity and time-averaged sediment concentration, cf. Eq. (5):

$$
q_{s}=\int_{\delta_{c}}^{h} \bar{u}(z) \cdot \bar{c}(z) d z
$$


where the time-averaged concentration is obtained from a conventional relationship, e.g. that by Ribberink and Al-Salem (1994):

$$
\bar{c}(z)=\bar{c}\left(z=\delta_{c}\right)\left(\frac{\delta_{c}}{z}\right)^{\alpha},
$$

in which the concentration $\bar{c}\left(z=\delta_{c}\right)$ is found from the solution of the contact load layer.

The concentration decay parameter $\alpha$ is an unknown value which has to be determined, e.g. from experiments. In general, it lies in the range from 1.5 to 2.1 (Ostrowski 2004), but in some cases much smaller values have been found correct, e.g. $\alpha=0.6$ (Biegowski 2005). For fine sand, the parameter $\alpha$ plays an important role in the modelling of sediment transport, as much of this transport takes place in suspension high above the sea bottom, particularly in the surf zone under severe storm conditions. Therefore, assumption of various $\alpha$ values yields a considerable spread of transport rates.

As already mentioned, the three-layer sediment transport model of Kaczmarek (1999), capable of dealing with joint wave-current conditions, makes use of the effective roughness height, representing the overall roughness of the sand grains building the bottom and the effects of bedload. This approach is particularly useful for the surf zone and intensive hydrodynamic wave-current impact under which the so-called sheet flow of sediments takes place and the sea bed forms are absent. Under milder wave conditions near the shoreline or during storms further from the shore (at a bigger depth), lower hydrodynamic regimes occur. They are accompanied by bed ripples, and no sheet flow can therefore be expected.

Recently, Ostrowski and Stella (2016) have presented a study concerning sediment transport beyond the surf zone under waves and wind-induced currents of the non-tidal sea. Their investigations were based on wave data collected at CRS Lubiatowo and a long-term reconstruction of the regional wind climate. In order to check the possibility of sediment motion at a depth of $18 \mathrm{~m}$, bed shear stresses were calculated for the following cases:

a) wave-induced nearbed oscillatory flow with the bottom roughness of sand grains (so-called "skin" bed roughness, as defined by Nielsen 2009);

b) wave-induced nearbed oscillatory flow with the overall bottom roughness (effective moveable bed roughness, as defined by Kaczmarek 1999);

c) nearbed stationary flow caused by the wind-driven current, with the "skin" bed roughness and the roughness resulting from the presence of bed forms of various heights;

d) nearbed flow caused by the wind-driven current superimposed on wave-induced nearbed oscillations, with the overall (effective) bottom roughness.

If the wave motion is superimposed on the stationary flow, an additional hydrodynamic effect appears, namely the apparent bottom roughness: the flow above the wave 
boundary layer encounters larger roughness due to the occurrence of waves (see e.g. Nielsen 2009). This effect was taken into account in the study of Ostrowski and Stella (2016). The modelling results showed that the highest bed shear stresses are generated by the joint action of waves and the wind-driven current. Further, it appears that the nonlinear wave-current interaction generates bed shear stresses bigger than would result from the superposition of results obtained separately for the impacts of waves and currents. Under extreme storm conditions, the calculated bed shear stresses are high enough to generate intensive sediment transport at the considered offshore location (18 $\mathrm{m}$ depth). Thus, it appears that in predicting sea bed lithodynamics beyond the surf zone it can be crucially important to take into account the wind-driven current.

It should be pointed out that a variety of bed shear stresses is obtained for various values of the geometrical bed roughness assumed in the modelling. Obviously, for a rippled sea bottom, the shear stresses increase with increasing roughness. This roughness is related to the height of the bed forms, but one cannot predict the bottom relief under given model conditions.

\section{Other Biases}

Many widely used methods for investigating the state and changes of a coast may prove inapplicable due to the presence of high erosion-resistance rocks at the seabed. Most of the classical coastal engineering approaches cannot be applied in studies of regions where the dynamic layer of non-cohesive sediments is lacking or very thin. Sediment transport models are based on the assumption that the supply of sea bed sediments is unlimited. These models make it possible to determine the sediment transport ability resulting from wave-current forcing. In the absence of sand in the coastal zone, actual sediment transport rates (particularly in long time scales) are definitely smaller than the potential rates yielded by theoretical models, irrespective of their character and background. To conclude, the computationally obtained sediment transport rate should be interpreted as the maximum volume of sand that can be displaced along the shore under given coastal hydrodynamic conditions. In other words, the sediment transport rate, determined conventionally on the basis of bed shear stresses generated by waves and currents, is merely the transport ability, the actual usefulness of which depends on the amount of sandy sediments accumulated in the nearshore dynamic layer.

As already mentioned, actual (natural) marine hydrodynamic processes are irregular (random). Although Kaczmarek and Ostrowski (1996) deduced that the sediment transport rates can be estimated for the irregular wave input represented by $H_{r m s}$ and $T_{p}$, one should be aware that precision in determining the bed roughness is somehow lost. For instance, the nearbed flow oscillations induced by the highest waves in the wave spectrum can "shave off" the bottom ripples so that the water flow takes place over a flat bed, not a bed with ripples formed by the "representative" wave conditions. 
Consequently, a false value of the bottom roughness can be assumed, which yields inaccuracy in the bed shear stresses determined.

At the most, coastal lithodynamics have been investigated theoretically using models of non-graded sediment. In such approaches, the motion of natural sand is modelled using the representative grain size. This is basically the median grain diameter $d_{50}$, but in some models other characteristic diameters of the grain size distribution are used, for instance $d_{90}$, see e.g. Van Rijn (1993). In a few theoretical approaches, however, more and more attention has been focused on the modelling of size-graded sediments. This direction of research was followed in modelling activities at IBW PAN, see e.g. Kaczmarek et al (2004) and Ostrowski (2004). It should be recommended to continue developing transport models for size-graded sand.

Coastal dynamics comprises both sea-borne and land-borne driving forces. As an example of the land-borne impact, one can mention the outflow of groundwater at the seaward slope of a dune or cliff, at its toe, at the backshore (emerged part of the beach) and even at the foreshore (permanently submerged part of the beach). These phenomenon is often underestimated by coastal engineers, while in the opinion of specialists in hydrogeology and soil mechanics it is the predominating abrasive factor at many locations of the Polish coast (particularly at some currently eroded cliffs). Other erosive impacts of non-sea origin are the rain wash on the cliff/dune face, the leakiness of wastewater systems and various destructive human activities (e.g. devastation of vegetation cover for recreational and tourist purposes). All these factors need to be studied thoroughly and, together with conventional coastal research, should constitute an efficient multidisciplinary approach.

\section{Final Remarks and Conclusions}

Although there has recently been great progress in description of the coastal wave-current velocity field and the mechanics of sediment transport, further investigations are necessary. In future, these investigations can ultimately yield a powerful quasi-phase-resolving or fully phase-resolving 3D computational framework, very reliable in solving practical problems encountered in coastal engineering. One should be aware that this will be a very tough challenge for coastal researchers. An attempt at modelling three-dimensional hydro- and morphodynamics has already been made, e.g. under the EU-COAST3D project, but it has been discovered (see Van Rijn et al 2003) that inaccuracies in 3D conditions are much larger than those in a pure 2D case because of the presence of 3D effects (e.g. rip currents). Further, it has been concluded that many nearshore processes cannot be simulated using the profile models. For instance, the 3D beach recovery processes in a post-storm period cannot be modelled at all, because these essentially $3 \mathrm{D}$ processes are not sufficiently known. Finally, as described in the present paper, the accuracy of the currently available cross-shore profile models greatly depends on proper calibration and tuning at all stages of simu- 
lation (from wave transformation to sediment transport), especially in computations for longer time scales (weeks, months).

The other modelling biases discussed in the present paper are related to approximations and simplifications concerning sediment resources, the random nature of actual marine hydrodynamic processes, the non-uniformity of sand grains building the sea bed, multi-aspect uncertainties in determination of bottom roughness and the impact of land-borne lithodynamic effects.

\section{Acknowledgements}

The study was sponsored by the Ministry of Science and Higher Education, Poland under mission-related programme No. 2 of IBW PAN.

\section{References}

Battjes J. A., Janssen J. P. F. M. (1978) Energy loss and set-up due to breaking of random waves, Proc. $16^{\text {th }}$ ICCE, Vol. I, 569-587.

Battjes J. A., Stive M. J. F. (1985) Calibration and verification of a dissipation model for random breaking waves, Journal of Geophysical Research, 90 (C5), 9159-9167.

Biegowski J. (2005) Dynamics of graded marine sediments in view of theory and experiment, $\mathrm{PhD}$ Thesis, IBW PAN Publishers, Gdańsk, 123 pp. (in Polish).

Fredsøe J. (1984) Turbulent boundary layer in combined wave-current motion, J. Hydraulic Eng., ASCE, 110 (HY8), 1103-1120.

Hsu T. W., Lan Y. J., Ostrowski R., Szmytkiewicz M. (2016) Wave Transformation in a Multi-Bar Surf Zone: Case Study of Lubiatowo (Poland), Archives of Hydro-Engineering and Environmental Mechanics, 63 (1), 19-34.

Kaczmarek L. M. (1999) Moveable Sea Bed Boundary Layer and Mechanics of Sediment Transport, DSc Thesis, IBW PAN, Gdańsk, ISBN 83-85708-35-9, 209 pp.

Kaczmarek L. M., Ostrowski R. (1996) Bedload Under Asymmetric and Irregular Waves: Theory Versus Laboratory Data, Archives of Hydro-Engineering and Environmental Mechanics, 43 (1-4), 21-42.

Kaczmarek L. M., Ostrowski R. (2002) Modelling intensive near-bed sand transport under wave-current flow versus laboratory and field data, Coastal Engineering, Elsevier Science B.V., 45 (1), 1-18.

Kaczmarek L. M., Biegowski J., Ostrowski R. (2004) Modelling cross-shore intensive sand transport and changes of bed grain size distribution versus field data, Coastal Engineering, Elsevier Science B.V., 51 (5-6), 501-529.

Nielsen P. (2009) Coastal and Estuarine Processes, Advanced Series on Ocean Engineering - Volume 29, World Scientific Publishing Co. Pte. Ltd., 343 pp.

Ostrowski R. (2004) Morphodynamics of a Multi-Bar Coastal Zone, DSc Thesis, IBW PAN Publishers, Gdańsk, ISBN 83-85708-64-2, 163 pp.

Ostrowski R., Stella M. (2016) Sediment Transport Beyond the Surf Zone Under Waves and Currents of the Non-Tidal Sea: Lubiatowo (Poland) Case Study, Archives of Hydro-Engineering and Environmental Mechanics, 63 (1), 3-17.

Ribberink J. S., Al-Salem A. (1994) Sediment transport in oscillatory boundary layers in cases of rippled beds and sheet flow, Journal Geoph. Res., 99 (C6), 12707-12727.

Roelvink D., Reniers A. (2012) A Guide to Modeling Coastal Morphology, Advances in Coastal and Ocean Engineering, Volume 12, World Scientific Publishing Co. Pte. Ltd., 274 pp.

Shore Protection Manual (1984) US Army Coastal Eng. Research Center. 
Svendsen I. A. (1984) Mass flux and undertow in a surf zone, Coastal Engineering, Elsevier Science B.V., 8, 347-365.

Szmytkiewicz M. (1996) 2D velocity distributions in nearshore currents, Proc. Coastal Dynamics '95, ASCE, New York, 366-376.

Szmytkiewicz M. (2002a) Wave-induced currents in the coastal zone, IBW PAN Publishers, Gdańsk, ISBN 83-85708-45-6, 235 pp. (in Polish).

Szmytkiewicz M. (2002b) Quasi 3D model of wave-induced currents in coastal zone, Archives of Hydro-Engineering and Environmental Mechanics, 49 (1), 57-81.

Van Rijn L. C. (1993) Principles of sediment transport in rivers, estuaries and coastal seas, Aqua Publications, Amsterdam, 614 pp.

Van Rijn L. C., Walstra D. J. R., Grasmeijer B., Sutherland J., Pan S., Sierra J. P. (2003) The predictability of cross-shore bed evolution of sandy beaches at the time scale of storms and seasons using process-based Profile models, Coastal Engineering, Elsevier Science B.V., 47, 295-327. 